\title{
Menaquinone Determination in the Taxonomy of Micrococcaceae
}

\author{
By L. JEFFRIES, M. A. CAWTHORNE, MARGARET HARRIS, \\ BARBARA COOK AND A. T. DIPLOCK \\ Beecham Research Laboratories, Vitamins Research Station, \\ Walton Oaks, Tadworth, Surrey
}

(Accepted for publication 29 July 1968)

SUMMARY

Staphylococci, micrococci and strains intermediate in guanine + cytosine (GC) content between these genera were all found to contain menaquinones. Two different types of menaquinone, described as 'normal' and 'hydrogenated' were revealed. Distinct and stable menaquinone patterns, formed by the percentages of individual isoprenologues, were found to characterize certain previously proposed species, groups or subgroups within the family. Studies on the distribution of menaquinones have also demonstrated heterogeneity within certain subgroups, notably Baird-Parker's Micrococcus subgroup 7, and the divisions within the yellow-pigmented micrococci revealed by Rosypal, Rosypalová \& Hołejs have been substantiated. Normal menaquinones were found in strains extending over a wide range of reported GC ratios $(3 \mathrm{I} \cdot 0$ to $6 \mathrm{I} \cdot 4 \%)$. These strains included staphylococci and non-pigmented micrococci, classified according to Baird-Parker, and also marine strains and others of uncertain taxonomic position. Hydrogenated menaquinones, however, were restricted to pigmented micrococci within the range $66 \cdot 3$ to $73.3 \%$ GC. There is thus evidence of a correlation between pigmentation, high GC ratio and menaquinone type. Menaquinone assay may contribute to the classification of Micrococcaceae and thus complement existing techniques in chemotaxonomy.

\section{INTRODUCTION}

Menaquinones, vitamins of the $\mathrm{K}_{2}$ group, are 2-methyl-I,4-naphthoquinones with an unsaturated polyisoprenoid side chain (MK); partially hydrogenated variants also occur in which at least one isoprene unit is saturated $(\mathrm{MK}(\mathrm{H}))$. Isoprenologues are designated by the number of isoprene units ( $\mathrm{n}$ ) in the side chain (Fig. I). Mena-

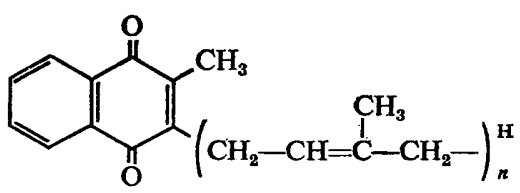

Fig. I. Menaquinones, vitamins of the $K_{2}$ group, are 2-methyl-r,4-naphthoquinones with unsaturated polyisoprenoid side chains; $n=$ number of isoprene units.

quinones have been extracted from both Gram-positive and Gram-negative aerobic bacteria (review by Pennock, 1966) and also from Bacteroides and Veillonella species (Gibbons \& Engle, I964); one isoprenologue, MK-4, has been isolated from animal 
tissues (Martius \& Esser, 1959) and also from a strain of Staphylococcus aureus (Cawthorne et al. 1967). Earlier investigations (Jeffries et al. 1967a) revealed the presence of menaquinones in all strains of staphylococci and micrococci examined. Several different menaquinone (MK) patterns, formed by different percentages of individual isoprenologues, were found and it was suggested that distinct MK patterns characterize $S$. aureus and certain accepted Micrococcus species. Subsequent studies of further strains of micrococci confirmed the taxonomic significance of their MK patterns (Jeffries et al. 1967b). The present paper describes our extended examination of aerobic Micrococcaeae and includes representatives of the genera Staphylococcus and Micrococcus, together with a number of mostly marine strains of intermediate and, at present, uncertain classification. Reported figures for guanine + cytosine content (GC ratio) in the DNA of the strains studied ranged from $31 \cdot 0$ to $74.0 \%$.

Baird-Parker (1965) divided the aerobic catalase-positive, Gram-positive cocci into two groups. Strains fermenting glucose were placed in group I (Staphylococcus) and those attacking glucose oxidatively, or not at all, in group 2 (Micrococcus). On physiological characters, Baird-Parker further divided the staphylococci into six subgroups, subgroup I representing $S$. aureus; the micrococci fell into eight subgroups, two of which corresponded to the accepted species Micrococcus luteus and M. roseus. More recently, Rosypal, Rosypalová \& Hořejš (1966), in studies mainly on pigmented micrococci, proposed a classification into groups based on the GC content of their DNAs; the strains were further divided into subgroups on similarity of biochemical and physiological characters. They proposed that, pending further data on the GC content of species within the Micrococcaceae, strains of GC content within the range 30.7 to $36.4 \%$ should be classified as Staphylococcus species and that only strains of GC content within the range $66 \cdot 3$ to $73 \cdot 3 \%$ should, at present, be accepted in the genus Micrococcus; attention was also drawn to a strain intermediate in GC content between these two genera. On DNA base composition Boháček, Kocur \& Martinec (1967a) divided a number of strains previously classified as micrococci into three groups. The first group comprised strains with GC ratio of $63.5-73.0 \%$ and included $M$. luteus and $M$. roseus, the second group consisted mainly of strains requiring reclassification, since they differed in GC content and also in other characters from the suggested ranges for the genera Micrococcus and Staphylococcus. Their third group consisted of motile strains, probably of marine origin, which were intermediate in GC content between the ranges for the genera Staphylococcus and Micrococcus, and it was suggested that these strains may be transferred to the genus Planococcus. Kocur \& Martinec (1965) proposed that the genus Sarcina should include only the anaerobic species with fermentative metabolism and that the species Sarcina lutea should be considered identical with $M$. luteus. Canale-Parola, Mandel \& Kupfer (1967) showed that the strict anaerobes, Sarcina ventriculi and Sarcina maxima, are distinct from aerobic micrococci in DNA base composition.

\section{METHODS}

Strains and cultivation. The strains studied included certain of those examined by Baird-Parker (1965), by Rosypal et al. (1966) and additional strains received from the Czechoslovak Collection of Micro-organisms (Brno), the Staphylococcus Reference Laboratory (Central Public Health Laboratory, London), and other sources (Table I). 
On receipt, all cultures were examined for purity and pigment production on nutrient agar at $26^{\circ}, 30^{\circ}$ and $37^{\circ}$ after incubation for I week; agar-slope subcultures were then stored at room temperature or at $4^{\circ}$. With the exception of Micrococcus morrhuae (see below), Lemco agar (blood agar base, Oxoid Ltd.) was found to be suitable for the cultivation of all strains, including those of marine origin. Nutrient agar plates inoculated with peptone-water suspensions of growth from freshly prepared agar-slope cultures were incubated for $48 \mathrm{hr}$ and the surface growth harvested for MK assay. Staphylococci were grown at $37^{\circ}$ and micrococci at $30^{\circ}$; in strains growing well at $30^{\circ}$ and $37^{\circ}$ the MK patterns were found to be similar at both temperatures. With most strains between I and $3 \mathrm{~g}$. wet weight of organism were extracted, requiring the inoculation of between 10 and 30 Petri dishes; with strains yielding only small amounts of MK quantities up to $9 \mathrm{~g}$. wet wt organism were prepared. The extreme halophile, $M$. morrhuae, did not grow on the nutrient agar and a medium of high salt concentration (Payne, Sehgal \& Gibbons, 1960) was used for maintenance of cultures and preparation of suspensions for extraction. Thirty plates inoculated from each strain of M. morrhuae were incubated at $37^{\circ}$ for 3 weeks in sealed tins containing flasks of water to maintain high humidity.

\section{Menaquinone assay}

Extraction of lipids. Since menaquinones are light-sensitive all operations were done in subdued light. The bacterial growth scraped from the surface of the medium was dispersed in ethanol ( $5 \mathrm{ml}$.) to remove water. The dispersion was centrifuged ( $1250 \mathrm{~g}$; $5 \mathrm{~min}$.) and the supernatant liquor decanted; ethanol ( $15 \mathrm{ml}$.), containing $50 \mathrm{mg}$. pyrogallol as antioxidant, was added to the residue and the mixture then boiled under reflux for $30 \mathrm{~min}$.; after cooling the supernatant fluid was removed. Water $(25 \mathrm{ml}$.) was added to the combined ethanolic liquors, which were then extracted with light petroleum (b.p. 40 to $60^{\circ}, 3 \times 25 \mathrm{ml}$.). This extract (extract A) was then evaporated and dried by azeotropic distillation under reduced pressure with benzene+ethanol $(\mathrm{I}+\mathrm{I}, \mathrm{V} / \mathrm{V})$. To test the efficiency of ethanolic extraction the residue remaining from this process was further subjected to alkaline digestion. Since this procedure destroys some MK (Jeffries et al. I967a) quantitative results must be treated with caution; however, we have no evidence that this results in changes in the isoprenologue pattern. The residue remaining from each I $g$. wet wt of extracted organisms was dispersed in $4 \mathrm{ml}$. ethanolic pyrogallol (pyrogallol $5 \%, w / v$, in ethanol, prepared freshly each day); 2 ml. aqueous potassium hydroxide solution ( $\mathrm{KOH}, 88 \mathrm{~g}$.; water $54 \mathrm{ml}$.) was then added and the mixture boiled under reflux for $30 \mathrm{~min}$. After cooling and the addition of water (Io $\mathrm{ml}$.) the lipid was extracted with ether $(3 \times 25 \mathrm{ml}$.). The ether extracts were pooled, washed free from alkali with water and evaporated; the lipid extract (extract B) was then dried.

Thin layer chromatography. Extracts A and B were treated identically, but separately. Each extract was dissolved in a small volume of light petroleum and applied as a streak to a $20 \times 10 \mathrm{~cm}$. plate coated with a $300 \mu$ layer of silica gel (Merck, A.G., Darmstadt) containing sodium fluorescein (50 p.p.m.). A spot of a pure MK solution was placed beside the streak to act as a marker and the chromatoplate was developed with benzene +light petroleum $(\mathrm{I}+\mathrm{I}, \mathrm{V} / \mathrm{v}$ ) to a distance of $8 \cdot 5 \mathrm{~cm}$. Menaquinones quench the green fluorescence when the plates are viewed under ultraviolet light, and thus appear purple against a green background; a marker of pure $\mathrm{MK}\left(R_{F} 0.4\right.$ in this 


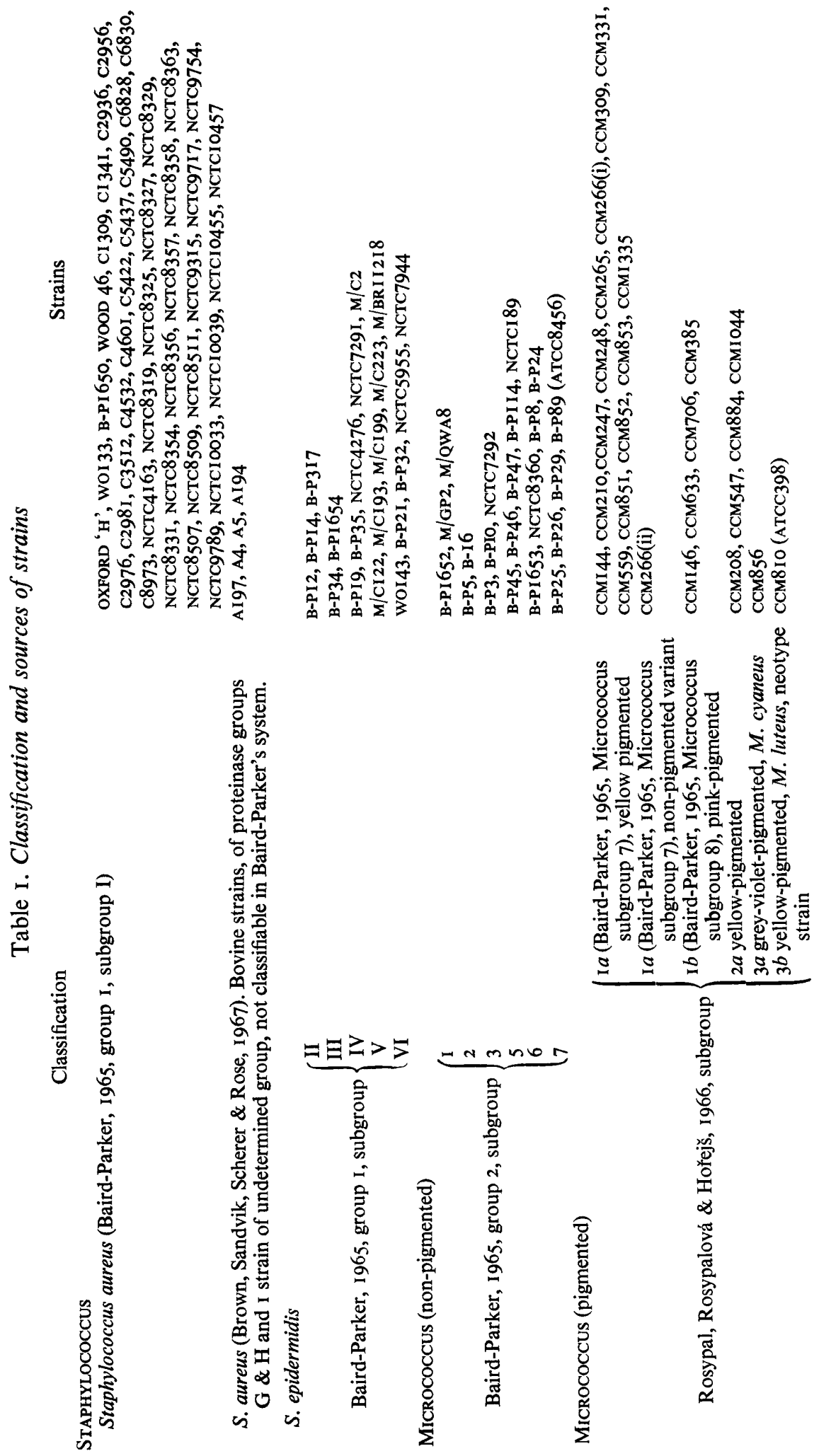




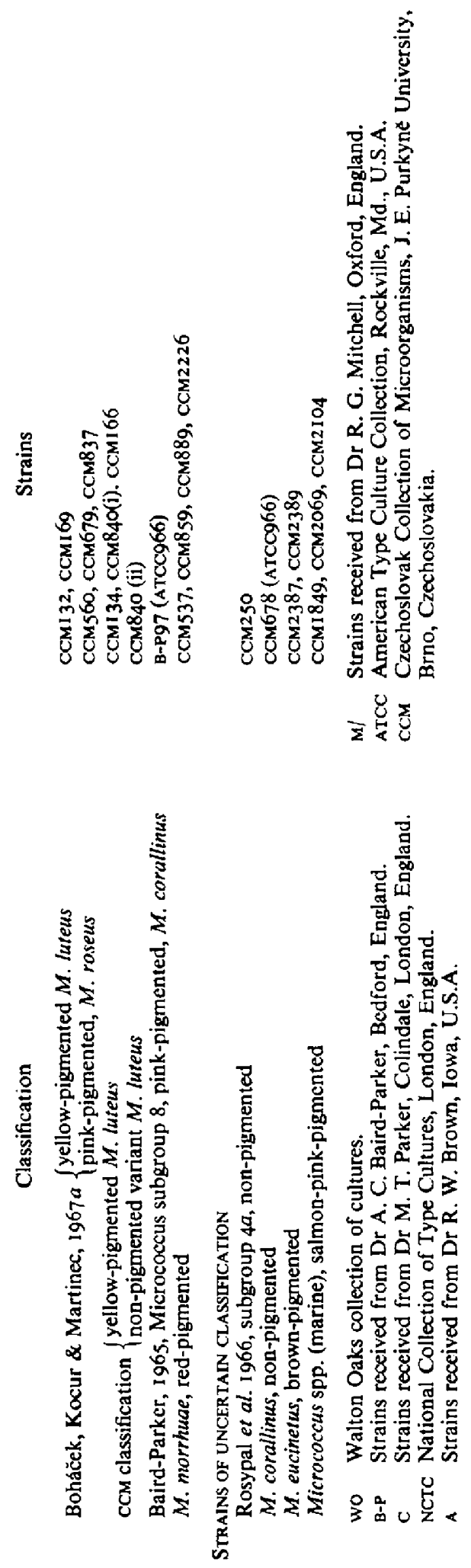


system) also aided identification. The MK band was scraped from the plate and extracted from the silica gel with ether vapour.

Reversed phase paper chromatography. Zinc-ammine solution was prepared by dissolving zinc oxide ( $16 \mathrm{~g}$.) and ammonium carbonate $(25 \mathrm{~g}$.) in a mixture of ammonium hydroxide (150 ml.) and water $(600 \mathrm{ml}$.); $20 \mathrm{ml}$. of $0.1 \%(\mathrm{w} / \mathrm{v})$ aqueous fluorescein solution was then added. Sheets of Whatman no. 4 grade 'chromatography paper', $23 \times 57 \mathrm{~cm}$., were immersed in the zinc ammine solution, hung to dry at room temperature and then baked in a fan-draught oven at 95 to $100^{\circ}$ for $\mathrm{I} \mathrm{hr}$. A portion of the sample was applied to a paper and partially overlapped with marker MKs. Since interfering substances were present the overlapping technique was necessary to ensure accurate identification of the MK isoprenologues. The remainder of the sample was applied to another paper, which was used for quantitative estimation of MKs. The phase of the papers was reversed by immersion in liquid paraffin solution $(3 \%$, w/v, solution liquid paraffin B.P. in light petroleum, b.p. 60-80 ). After evaporation of the light petroleum solvent the papers were developed in a filter-paper lined, vapour-saturated tank, by descending chromatography with $95 \%(\mathrm{v} / \mathrm{v})$ ethanol in water for $5 \mathrm{hr}$. at $27^{\circ}$. The papers were then dried with warm air and examined under ultraviolet light. Separation and migration of MKs in reversed-phase chromatography are related to isoprenoid side-chain length, thus MK-6 migrates farther than MK-7. After elution from the quantitative paper, the separate MK isoprenologues were measured.

Measurement and identification of isolated menaquinones. When examined by ultraviolet spectrometry menaquinones showed maxima at 239, 249, 260 and $270 \mathrm{~nm}$. The absorption at $285 \mathrm{~nm}$. was very small and the difference in absorption value $(\Delta E)$, observed at 285 and at $270 \mathrm{~nm}$., was used as the basis of the assay. It was assumed that irrelevant absorption over this short range would have been constant. $\Delta E_{1 \mathrm{~cm}}^{1 \%}$ values determined experimentally in our laboratories on synthetic MKs were as follows: MK-9, 222; MK-8, 238; MK-7, 244; MK-6, 272. To complete the identification of MKs a complete u.v. scan from 230 to $350 \mathrm{~nm}$. was made to ascertain that all four absorption maxima were present. Hydrogenated menaquinones, when present, ran more slowly than normal menaquinones on paper chromatography and thus occupied a position on the paper chromatogram between the normal isoprenologues.

\section{RESULTS}

The results of the menaquinone assays are shown in Tables $2 a$ and $b$. Table 3 indicates the correlation between representative values for GC content in DNA and the type of menaquinone produced.

Menaquinones identified as MK-6, MK-7, MK-8 and MK-9 (referred to as 'normal' MKs, designated MK-n, with $R_{F}$ values $0.318,0.213,0.143,0.100$, respectively) were found in staphylococci, micrococci and strains of intermediate uncertain classification. However, certain strains of micrococci with high $\mathrm{GC}$ values yielded menaquinone fractions which, when resolved in our paper chromatographic system, gave substances with $R_{F}$ values $0.275,0.182,0.124$ and 0.086 . These substances gave a u.v. spectrum typical of menaquinones. Calculation of their $R_{F}$ values suggested that they were partially hydrogenated MKs (referred to as 'hydrogenated' MKs and designated MK-n (H)). This conclusion was substantiated by their behaviour on thin layers of silver nitrate-impregnated silica gel. 


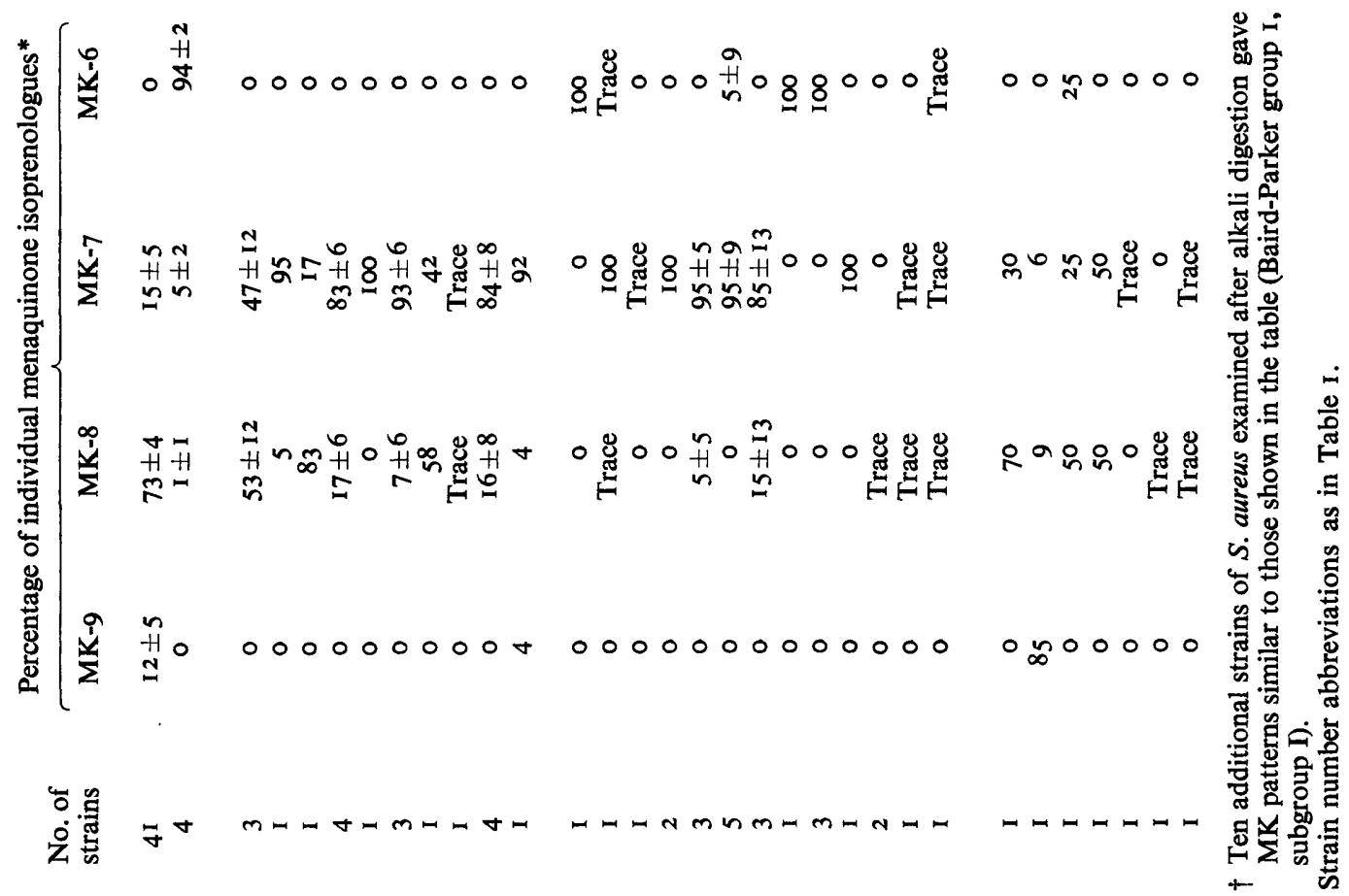

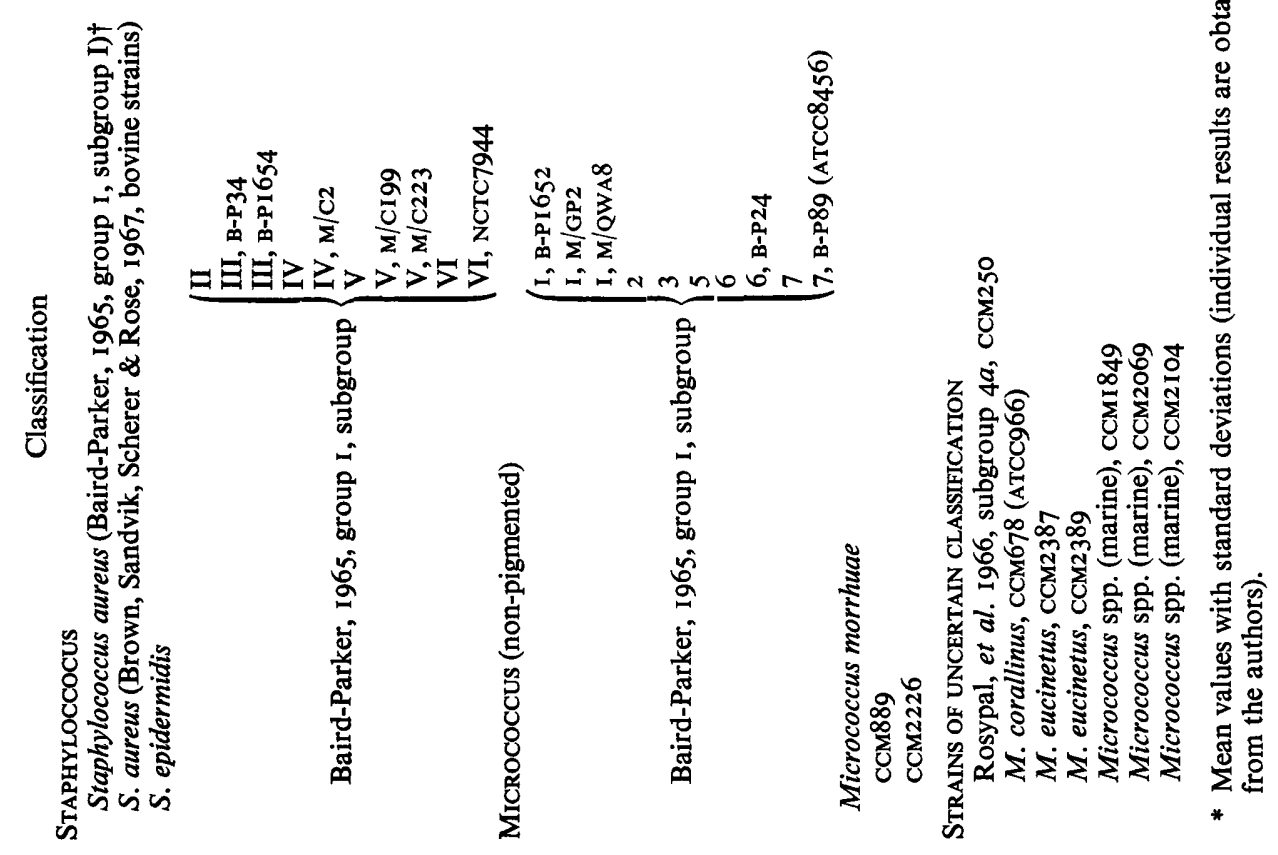




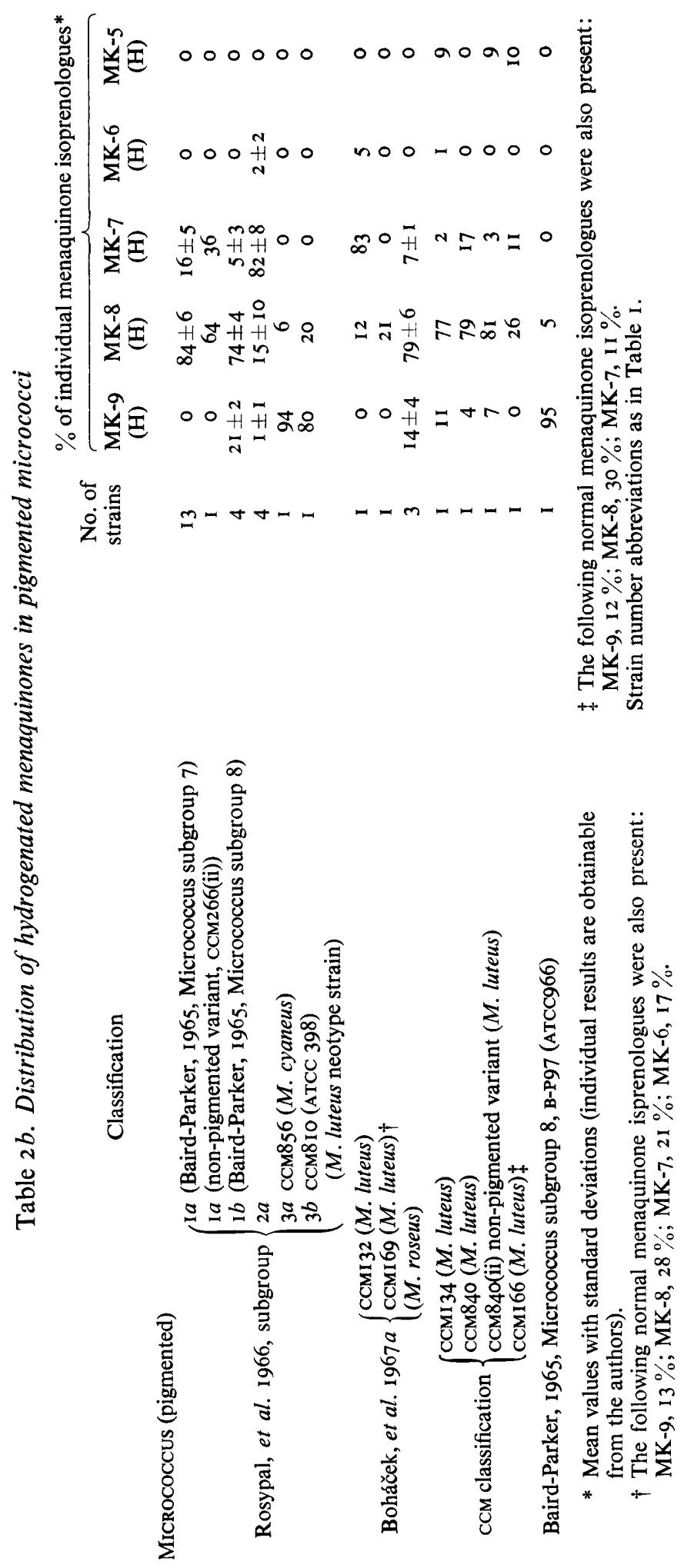




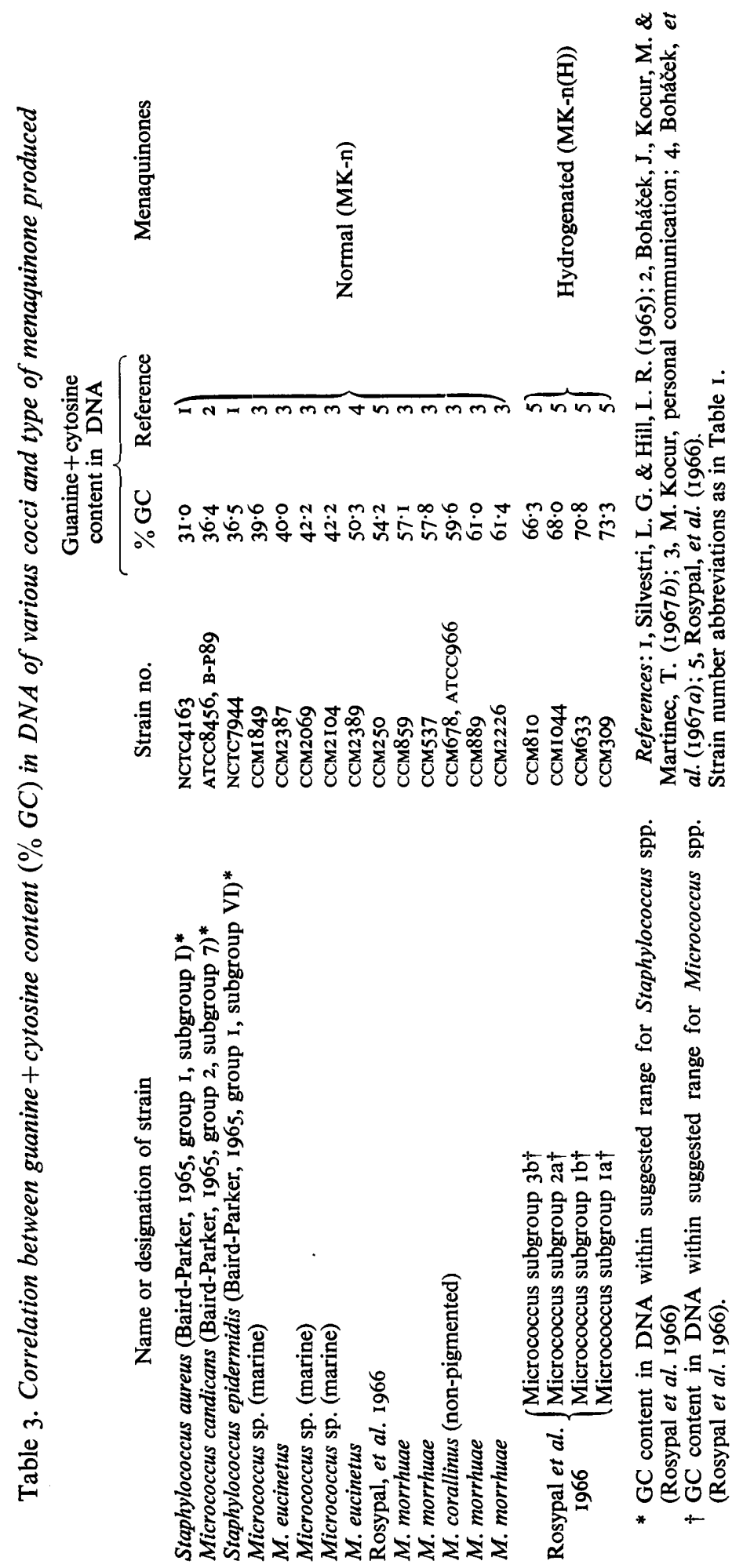


Although, for a given strain, the yield of MK was found to vary somewhat between cultures grown on different occasions, the isoprenologue ratios (MK patterns) were found to be constant. The reasons for this variation in MK yield are not known and our limited experiments yielded negative results. However, it has been shown, with a vitamin-K dependent strain of Fusiformis melaninogenicus, that MKs diffuse into agar from growing bacteria and this may represent a variable loss, since we only extracted the bacterial mass and not the culture fluid. The amounts of MK extracted ranged from a trace (less than I $\mu \mathrm{g}$.) to more than $400 \mu \mathrm{g}$./g. wet wt organism. In general, the highest yields were from the Staphylococcus aureus strains and the lowest were from the strains originally isolated from marine sources, including the extreme halophile, Micrococcus morrhuae (Table $2 a$ ). The MK patterns attributed to strains producing very small amounts of MK should be regarded as approximate, since the errors involved in such calculations may be considerable; but the identification of the type of MK produced by these strains was, in all cases, unequivocal.

\section{Menaquinone patterns}

As shown in Table $2 a$, MK-7 and MK-8 were common to both staphylococci and certain micrococci; with the exception of one strain of uncertain classification (CCM 678, ATcC966, discussed below), MK-9 was found only in staphylococci. Menaquinone-6, although present as the major isoprenologue in strains of a physiologically distinct group of coagulase-producing staphylococci, was not found in any other strains within this genus. Menaquinone- 6 was, however, present in certain nonpigmented micrococci, and in some strains it was the only isoprenologue detected. Hydrogenated MKs were restricted to yellow, pink and violet-pigmented strains (Micrococcus luteus, $M$. roseus, $M$. cyaneus) and the non-pigmented variant colonies derived from two $M$. luteus strains (Table $2 b$ ). As discussed below, normal MKs were present in strains ranging widely in GC content, whereas hydrogenated MKs were extracted only from strains within the highest GC range.

The MK patterns of all strains have remained stable after repeated subculture and also after freeze-drying.

\section{DISCUSSION}

\section{Staphylococcus}

Staphylococcus aureus. With the exception of a single culture, described below, all the coagulase-producing strains comprising the accepted species, $S$. aureus, classifiable in group I, subgroup I of Baird-Parker (I965) showed a single and characteristic MK pattern, with MK-8 as the major isoprenologue. MK-9 and MK-7 were present as minor isoprenologues in all strains, but their relative proportions differed between strains. The strains studied varied widely in sensitivity to antibiotics and in bacteriophage type; they included the twenty-one propagating strains of the International Phage Typing series.

Four cultures of Staphylococcus aureus NCTC75I I were received from different laboratories; this strain has been widely distributed as propagating strain 53 of the International Phage Typing series. Three of the cultures gave the MK pattern characteristic of $S$. aureus Baird-Parker group I subgroup I as shown in Table $2 a$. One culture, however, which had been employed in genetical studies by Korman (1963), revealed an anomalous MK pattern (MK-8, 17\%; MK-7, 48\%; MK-6, I I \%; 
MK-5, I5\%; MK-4, $9 \%$ ) including two isoprenologues, MK-4 and MK-5, not previously encountered in a bacterium. This culture was indistinguishable, in bacteriophage studies, from the other three cultures of NCTC85II. Detailed studies on the menaquinones of these cultures of NCTC85I I, and on mutants allegedly derived from one of them, were described by Jeffries, Harris \& Price (I967c). Identical GC ratios $(32.4 \%)$ were found in the culture of NCTC85I I giving an anomalous MK pattern and in one of the cultures of this strain from another source (L. R. Hill, personal communication).

Four bovine strains, isolated by Brown, Sandvik, Scherer \& Rose (1967), were coagulase and phosphatase positive but did not produce acetoin from glucose or ferment maltose or mannitol. These strains, with features common to both Staphylococcus aureus (Baird-Parker group I subgroup I) and S. epidermidis (Baird-Parker group I subgroup III), were therefore not classifiable in Baird-Parker's system; they were, moreover, clearly separable on MK pattern from the strains of $S$. aureus studied by Baird-Parker (1965), all of which fell into his subgroup I.

Staphylococcus epidermidis. Of the twenty strains classified in Baird-Parker's subgroups II to VI, all except one of the subgroup IV strains, which contained MK-7 only, produced both MK-7 and MK-8. Two strains (NCTC729I, 7944), one in subgroup IV and one in subgroup VI, also produced small amounts of MK-9; MK-6 was not detected in any of the $S$. epidermidis strains. The strains could be broadly divided into two groups, according to the relative proportions of MK-7 and MK-8. The ratio of MK-7:MK-8: varied from about 0.5 to I0, or even higher; the highest ratios tending to predominate within subgroups IV, V and VI. A strain of the violet-pigmented Micrococcus violagabriellae (strain B-P3I 7 in Table I), now reclassified as a subgroup II Staphylococcus (Baird-Parker, 1965), showed an MK pattern similar to that of the two other strains of subgroup II staphylococci examined.

\section{Micrococcus}

Non-pigmented strains. The nineteen strains examined belonged to Baird-Parker's Micrococcus group 2, subgroups I, 2, 3, 5, 6 and 7; they produced normal MKs only. MK-7 was the major isoprenologue in most strains and in ten of them either MK-7 or MK-6 was the sole isoprenologue detected. Menaquinone- 8 was found, in relatively small amounts, in a few strains. Certain MK patterns were common to strains in different Baird-Parker subgroups. Strain NCTC8360, formerly classified as Staphylococcus aureus and alleged to clot sheep plasma, but not human or rabbit plasma (K. J. Steel, cited by Baird-Parker, 1965) was found by Baird-Parker to belong to Micrococcus subgroup 6. The MK pattern of this strain supports its separation from $S$. aureus.

Pigmented strains. In contrast to the non-pigmented strains, considered above, the yellow and pink-pigmented strains classified in Baird-Parker's subgroups 7 and 8 respectively, together with other pigmented strains classified by Rosypal et al. (I966), produced hydrogenated MKs (Table $2 b$ ). All except one of the pigmented strains B-P97, ATCC966) were from the Czechoslovak Collection of Micro-organisms and included representatives of the groups and subgroups proposed by Rosypal $e$ al. and a few of the strains studied by Boháček et al. (1967a).

Two species (Micrococcus luteus, $M$. roseus) are recognized in the genus Micrococcus (Evans, 1965) but, in the tests described by Baird-Parker (1966), pigment is the only character which reliably separates these species from each other, their separation from 
micrococci in the other subgroups being mainly on negative evidence. Differences in cell-wall composition and in the protein contents of the cocci are regarded as criteria justifying the separation of the species, although stable pink-pigmented mutants are alleged to have been produced from a yellow-pigmented strain classifiable in BairdParker subgroup 7 (G. W. Gould, cited by Baird-Parker, 1965).

Baird-Parker (1965) classified the yellow-pigmented Micrococcus luteus strains, together with non-pigmented strains, in Micrococcus subgroup 7, but our MK studies show that this subgroup is not homogeneous: of the strains examined by us all the yellow-pigmented ones contained hydrogenated $\mathrm{MKs}$, and the non-pigmented strains normal MKs. We suggest a division of these strains into two subgroups, based on type of menaquinone produced. This separation is further supported by the higher sensitivity of the yellow-pigmented strains to lysozyme (unpublished observations). Non-pigmented variants (CCM266 (ii); CCM840 (ii)) isolated in our laboratory from two yellow strains have, like the parent strains, contained hydrogenated MKs.

Rosypal et al. (1966) divided the yellow-pigmented Micrococcus strains into three groups on differences in GC content within the range $66 \cdot 3$ to $73.3 \%$; they found that strain ATCC398 proposed as the type strain of $M$. luteus by Breed (1952), was in a different group (3) from the other strains classified as $M$. luteus which they studied and placed in group I. These authors considered that, since the name of the type species should not be changed, the application of the specific name luteus to strains within their subgroup I $a$, corresponding to Micrococcus subgroup 7 of Baird-Parker, should be questioned. Although the significance of minor differences in GC content, such as those found by Rosypal et al., remains to be substantiated, a correlation has been found between the proposed subgroups of Rosypal et al. and MK patterns. Although the yellow-pigmented subgroup 2 a strains of Rosypal et al. were alleged to correspond to Micrococcus subgroups 5 and 6 of Baird-Parker, we were unable to confirm this finding; we found that, in the tests recommended by Baird-Parker, these strains more closely resembled those in subgroup 7 .

\section{Correlation between MK patterns and the classification of micrococci proposed by Rosypal et al. (1966)}

Subgroup $\mathrm{r} a$. Thirteen strains were tested; all were yellow and some produced violet pigment also. They gave a distinct $\mathrm{MK}$ pattern, with $\mathrm{MK}-8(\mathrm{H})$ as the main isoprenologue and $\mathrm{MK}-7(\mathrm{H})$ as minor; small amounts of $\mathrm{MK}-6(\mathrm{H})$ were found in some strains and one strain also produced a trace of MK-9(H). Strain CCM266 yielded unstable variants lacking pigment (226 (ii)); in MK pattern these were identical with the pigmented parent strain (266(i)).

Subgroup $I b$. The four strains examined produced pink pigment and revealed a single MK pattern, with $\mathrm{MK}-8(\mathrm{H})$ as major and $\mathrm{MK}-9(\mathrm{H})$ and $\mathrm{MK}-7(\mathrm{H})$ as minor isoprenologues. The presence of $\mathrm{MK}-9(\mathrm{H})$ and the lower relative percentage of MK- $7(\mathrm{H})$ differentiates this subgroup from the strains in subgroup I a; MK-8(H) as the main isoprenologue is common to both subgroups, and perhaps supports their suggested close relationship.

Subgroup 2 a. Four strains (two previously classified as Micrococcus varians and two as $M$. conglomeratus) showed a single $\mathrm{MK}$ pattern, in which $\mathrm{MK}-7(\mathrm{H})$ predominated, MK-8(H) was present in all strains and two of them also contained small amounts of MK-6(H) or MK-9(H). 
Subgroups $3 a$ and $3 b$. The single strains comprising these two subgroups gave a similar and distinct $\mathrm{MK}$ pattern in which $\mathrm{MK}-9(\mathrm{H})$ was the main isoprenologue and MK-8(H) was the minor. Micrococcus cyaneus (CCM856) represents subgroup $3 a$ and produces grey/violet rough colonies. It was regarded by Kocur \& Martinec (I963) as an atypical strain of $M$. luteus; Rosypalová et al. (1966), however, regarded this species as distinct. The type strain of $M$. luteus (ATCC398) represents subgroup $3 \mathrm{~b}$.

Subgroup 4a. The single strain in this subgroup (CCM250), differing in GC ratio (56.4\%) from staphylococci and micrococci, contained normal MKs only; the pattern (MK-8, 70\%; MK-7, 30\%) resembles that of certain coagulase-negative staphylococci.

The yellow-pigmented Micrococcus strains studied by Rosypal et al. (1966) fell into three ranges in GC content; each has now been shown to have a distinct MK pattern. Furthermore, the subgroup I a strains have been shown to be sensitive to lysozyme by a plate incorporation method, whereas the subgroup 2 a strains were relatively resistant (unpublished observations).

The menaquinone patterns of other yellow and pink-pigmented Microccoccus strains studied. Three pink-pigmented strains classified as $M$. roseus by Boháček et al. (1967a) gave an MK pattern identical with the strains of this species classified by Rosypal et al. (I966) in their subgroup I $b$, thus all seven $M$. roseus strains examined were homogeneous in MK pattern.

Two allegedly non-pigmented strains of Micrococcus luteus, unlike the thirteen strains of this species classified in subgroup I a of Rosypal et al. (1966), produced an MK pattern similar to that of the $M$. roseus strains. On nutrient agar at $30^{\circ}$ one of these strains (CCM840; GC 7I \%) produced numerous colonies lacking pigment, together with a single yellow colony; on subculture these colonies remained stable with respect to pigmentation. Although the MK patterns of the two colony types were similar, the non-pigmented variant produced an additional isoprenologue (tentatively identified as $\mathrm{MK}-5(\mathrm{H})$ ), which was not encountered in any of the other strains in our series. The presence of MKs of the hydrogenated type suggests that the non-pigmented variant colonies (СCM840, (ii)) unlike the non-pigmented strains in Baird-Parker subgroup 7 which produced normal MKs, arose as variants from an originally yellow-pigmented strain. The other strain (CCMI34), received as nonpigmented, produced a pale yellow pigment at $30^{\circ}$ which intensified at room temperature; the MK pattern of this strain was also like that of the $M$. roseus strains. A pink-pigmented strain (B-P97, ATCC966) of $M$. corallinus (Baird-Parker Micrococcus subgroup 8) showed an MK pattern similar to that of the type strain of $M$. luteus, classified in subgroup 3 of Rosypal et al. Menaquinone patterns may thus substantiate the suggested close relationship between the yellow and pink-pigmented strains at present accorded specific status.

Two yellow-pigmented Micrococcus luteus strains (CCMI69, GC 73.3\%; CCMI66, GC $73.4 \%$ ) each contained normal and hydrogenated menaquinones; we have not encountered a mixture of normal and hydrogenated meanquinones in any of the other strains examined. "However, it should be stressed that hydrogenated menaquinones have been found to be characteristic of pigmented micrococci and have not been detected in any other strains. 


\section{Strains of uncertain classification}

Strains originally described as Micrococcus species, and subsequently found to be of intermediate position in GC content ( 48 to $5 \mathrm{I} \%$ ) between the suggsted ranges for the genera Staphylococcus (30.7 to $36.5 \%$ ) and Micrococcus $(66.3$ to $73.3 \%$ ) were described by Boháček et al. (1967a). These authors suggested that certain motile strains, including the light-brown pigmented $M$. eucinetus should, in view of their $\%$ GC, motility and marine habitat, be transferred to the genus Planococcus. The two strains of $M$. eucinetus examined (GC 40 and $50.3 \%$ ) each produced less than $20 \mu \mathrm{g}$. MK of the normal type/g. wet wt organism. Three other strains of salmonpink pigmented marine micrococci (CCMI849, 2069 and 2104) were received from Dr M. Kocur, together with estimates of their GC content (39.6 to $42 \cdot 2 \%$ ); these strains also yielded normal MKs, but in insufficient amounts for quantitative estimation of individual isoprenologues. Normal MKs (mainly MK-8) were found in very small quantities, necessitating the extraction of between 7 and $9 \mathrm{~g}$. wet wt organism, in four strains of the red-pigmented $M$. morrhuae, grown for 3 weeks on a special medium of high salt content formulated for halophilic cocci; a fifth strain (CCM2224) grew relatively poorly, yielding only $2.4 \mathrm{~g}$. wet wt organism, from which we were unable to extract sufficient MK for characterization. The GC content of the strains of $M$. morrhuae ranged from $57^{\cdot} \mathrm{I}$ to $6 \mathrm{I} \cdot 4 \%$ (M. Kocur, personal communication).

A strain submitted as a non-pigmented variant of Micrococcus corallinus (ССM678, ATCC966) was classified by us on negative characters and placed in Baird-Parker's Micrococcus subgroup 7. This strain, with a reported GC ratio of $59.6 \%$ (M. Kocur, personal communication), has a value somewhat lower than those within the range for pigmented Micrococcus strains. This strain produced small quantities of menaquinones of the normal type but, as shown in Table $2 a$, the MK pattern, with MK-9 as major isoprenologue, was not encountered in any other strain that we examined. The GC ratio of the pink-pigmented strain, ATCC966, B-P97, has not been determined, but the MK pattern, with hydrogenated isoprenologues (Table $2 \mathrm{~b}$ ) suggests that the alleged non-pigmented variant strain is not related to it.

The strain with $56.4 \%$ GC in DNA, representing subgroup 4 a of Rosypal et al. (1966) discussed above, like the other strains intermediate in position between micrococci and staphylococci, also yielded normal MKs.

Strains NCTC7292 and NCTCI89 were received as Staphylococcus saprophyticus and S. lactis, respectively. Both strains were submitted to Dr A. C. Baird-Parker, who classified them as micrococci (NCTC7292 in Micrococcus subgroup 3; NCTC89 in Micrococcus subgroup 5). In the standard medium for the determination of anaerobic utilisation of glucose with a paraffin oil seal, proposed by the International Subcommittee on Staphylococci and Micrococci (1965), we found that NCTC7292 produced acid only at the top of the tube, and NCTCI89 did not produce acid. Mortensen \& Kocur (1967) confirmed that, although neither strain visibly fermented glucose in the proposed standard medium containing the indicator bromocresol purple, both strains formed acid weakly and slowly, as revealed by potentiometric $\mathrm{pH}$ determination of anaerobic cultures, in a fluid medium containing glucose. These two strains, together with others of different GC ratios, were thus found by Mortensen \& Kocur to be intermediate between strains rapidly fermenting glucose, all of which were of low GC ratio (staphylococci), and strains forming little if any acid from glucose by oxidation 
and all of high GC ratio (micrococci). The GC ratios of strains 7292 and I89 (Table I) suggest their classification with the genus Staphylococcus, but this is not supported by their relative resistance to novobiocin, determined according to Mitchell \& BairdParker (1967). The presence of MK-6 in both strains suggests that they may be closer to strains at present classified as non-pigmented micrococci.

Micrococcus candicans, ATCC8456, B-P89, was classified on physiological characters in Baird-Parkers' Micrococcus subgroup 7; however, the GC ratio of this strain, reported as $36.4 \%$ by Boháček, Kocur \& Martinec ( $1967 b)$, suggests that it should be classified in the genus Staphylococcus. As shown in Table $2 a$, all four non-pigmented subgroup 7 Micrococcus strains produced normal menaquinones; but strain ATCC8456 showed a different pattern from the other three strains, on which GC values have not been established.

It would appear, from the literature, that GC ratios have been determined on a disproportionately large number of strains of pigmented micrococci and on relatively few staphylococci and non-pigmented micrococci. A few non-pigmented strains, at present classified as micrococci on biochemical and physiological characters, have GC ratios which suggest that, in fact, these strains should not be classified in the genus Micrococcus ( A. C. Baird-Parker, personal communication).

\section{Association between menaquinone type and GC ratio}

In addition to the values shown in Table 3, GC ratios, which fell within the range 66 to $74 \%$, were provided for all the pigmented micrococci received from the Czechoslovak Collection of Micro-organisms. Thus 45 of the I29 strains of Micrococcaceae on which we have determined menaquinones were of known GC content.

According to the type of menaqinone produced, aerobic Micrococcaceae may be divided into two groups. The first, broader, group would include strains with GC ratios lower than $6 \mathrm{r} \cdot 4 \%$. The second group would comprise all strains producing hydrogenated menaquinones and would include those at present classified as Micrococcus luteus and M. roseus (\% GC 66 to 74). Within these groups, subgroups may be defined on MK patterns. It will be interesting to see whether further data on GC ratios supports the differences suggested by the menaquinone distribution.

We are grateful to Dr M. Kocur, Curator, Czechoslovak Collection of Microorganisms, who supplied the strains of marine cocci and most of the pigmented micrococci, together with GC values where they were not available from the literature. To Dr A. C. Baird-Parker we are indebted for providing strains representative of his proposed classification and also for many helpful discussions during the course of our work. For supplying other strains, together with details of isolation or classification, we are pleased to record our thanks to Mrs E. Asheshov, Dr M. T. Parker, Dr S. P. Lapage and Dr R. G. Mitchell. Dr O. Isler (Hoffman la Roche, Basle, Switzerland) kindly provided samples of synthetic menaquinones. For constructive criticism during the course of this work and in its presentation we thank Mr S. A. Price and Dr J. Green. 


\section{REFEREN CES}

BAIRD-PARKer, A. C. (1965). The classification of staphylococci and micrococci from world-wide sources. J. gen. Microbiol. 38, 363 .

BaIrd-PARKer, A. C. (I966). Identification Methods for Microbiologists, Part A, Ed. B. M. Gibbs \& F. A. Skinner. London: Academic Press.

BohÁČEK, J., Kocur, M. \& MARTINeC, T. (1967a). DNA base composition and taxonomy of some micrococci. J. gen. Microbiol. 46, 369.

BoHÁČEK, J., KOCUR, M. \& MARTINEC, T. (1967b). Deoxyribonucleic acid base composition of atypical micrococci. Reports from the Conference on the Taxonomy of Bacteria held in the Czechoslovak Collection of Micro-organisms, J. E. Purkyne University, Brno, 27-29 September 1967, Spisy prir. Fak. Univ. Brne. K 40 (483), 237 (1967/5).

BreEd, R. S. (1952). The type species of the genus Micrococcus, Int. Bull. bact. Nomencl. Taxon. 2, 85.

Brown, R. W., Sandvik, O., Scherer, R. K. \& Rose, D. L. (1967). Differentiation of strains of Staphylococcus epidermidis isolated from bovine udders, J. gen. Microbiol. 47, 273.

Canale-Parola, E., Mandel, M. \& Kupfer, D. G. (1967). The classification of Sarcinae, Arch. Mikrobiol. 58, 30.

Cawthorne, M. A., Jeffries, L. R., Harris, M., Price, S. A., Diplock, A. T. \& Green, J. (I967). Menaquinone-4 and -5 in a bacterium, Biochem. J. ro4, $35 \mathrm{C}$.

Evans, J. B. (1965). Current views and problems relating to the taxonomy of the Micrococcaceae. Int. Bull. bact. Nomencl. Taxon. 15, I I I.

GibBons, R. J. \& EnGLe, L. P. (1964). Vitamin K compounds in bacteria that are obligate anaerobes. Science, N.Y. 146, 1307.

International Subcommitee on Staphylococci AND Micrococci (1965). Recommendations of subcommittee. Int. Bull. bact. Nomencl. Taxon. I5, 109.

Jeffries, L., Cawthorne, M. A., Harris, M., Diplock, A. T., Green, J. \& Price, S. A. (i967a). Distribution of menaquinones in aerobic Micrococcaceae. Nature, Lond. 215, 257.

Jeffries, L., Cawthorne, M. A., Harris, M., Diplock, A. T., Green, J. \& Price, S. A. (i967b). Menaquinone patterns of Micrococcaceae: their possible taxonomic significance. Reports from the Conference on the Taxonomy of Bacteria held in the Czechoslovak Collection of Microorganisms, J. E. Purkynè University, Brno, 27-29 September 1967. Spisy prir. Fak. Univ. Brno. K 40 (483), 230, (1967/5).

Jeffries, L., HARris, M. \& Price, S. A. (1967c). Atypical menaquinone pattern in a strain of Staphylococcus aureus. Nature, Lond., 216, 808.

KocUR, M. \& MARTINEC, T. (1963). The classification of some violet-pigmented micrococci. J. gen. Microbiol. 32, 185.

KocUR, M. \& MARTINEC, T. (1965). Some remarks on the classification of micrococci. Int. Bull. bact. Nomencl. Taxon. 15, 113.

KORMAN, R. Z. (1963). Coagulase-negative mutants of Staphylococcus aureus; genetic studies, J. Bact. $86,363$.

MartiUs, C. \& EsSER, H. O. (1959). Constitution of the vitamin $\mathrm{K}$ formed by the animal organism from 2-methylnaphthoquinone, Biochem. Z. 33r, I.

Mrtchell, R. G. \& BAIRD-PARKer, A. C. (1967). Novobiocin resistance and the classification of staphylococci and micrococci, J. appl. Bact. 30, $25 \mathrm{I}$.

MORTENSEN, N. \& Kocur, M. (1967). Correlation of DNA base composition and acid formation from glucose of staphlococci and micrococci, Acta path. microbiol. scand. 69, 445.

Payne, J. I., Sehgal, S. N. \& Gibbons, N. E. (1960). Immersion refractometry of some halophilic bacteria. Can. J. Microbiol. 6, 9.

PENNOCK, J. F. (I966). Occurrence of Vitamin K and related quinones. Vitams. Horm. $24,307$.

Rosypal, S., Rosypalová, A. \& Hořejš, J. (I966). The classification of micrococci and staphylococci based on their DNA base composition and Adansonian analysis. J. gen. Microbiol. 44, 28I.

Rosypalová, A., BoháčEK, J. \& Rosypal, S. (1966). Deoxyribonucleic acid base composition and taxonomy of violet-pigmented cocci. Antonie van Leeuwenhoek 32, 105.

Silvestri, L. G. \& Hill, L. R. (1965). Agreement between deoxyribonucleic acid base composition and taxometric classification of gram-positive cocci. J. Bact. 9o, I36. 